Neurosurg Focus 13 (2):Article 6, 2002, Click here to return to Table of Contents

\title{
Effects of nerve root retraction in lumbar discectomy
}

\author{
Carlos Feltes, M.D., Kostas Fountas, M.D., Rostislav Davydov, M.D., \\ Vassilios Dimopoulos, M.D., and Joe Sam Robinson, JR., M.D. \\ Georgia Neurosurgical Institute, Medical Center of Central Georgia, Macon, Georgia
}

\begin{abstract}
The authors studied whether the amount of retraction pressure applied to a compromised nerve root during lumbar discectomy has an impact on intra- or postoperative outcome.

Methods. The authors conducted a prospective analysis of 20 patients. There were 12 men and 12 women whose mean age ( \pm standard deviation $[\mathrm{SD}]$ ) was 42.25 years \pm 15 years (range 21-65 years). During intraoperative electromyography (EMG) monitoring, measurements were obtained during routine retraction of the affected nerve root by using a specially designed and constructed nerve root retractor connected to a reconfigured personal computer for this specific purpose. Follow-up results were assessed in the immediate postoperative period and at up to 1 year. The maximum measured force applied during random periods of time was $9.85 \mathrm{~N} /$ second (mean $6.95 \pm \mathrm{N} / \mathrm{second}[ \pm \mathrm{SD}]$ ). The mean retraction time was $39.5 \pm 21$ (SD). No intraoperative EMG-detected irritation was noted during or after routine retraction. In four of 20 patients, sensory changes occurred at the ipsilateral nerve root level, which resolved at the time of discharge.

Conclusions. The authors found that routine nerve root retraction does not cause nerve root irritation, as demonstrated by EMG monitoring, nor was patient outcome affected in this series.
\end{abstract}

KEY WORDS • lumbar spine • discectomy • retraction • outcome

Despite the evolution of technology in neurosurgery, lumbar discectomy continues to be the most common operative procedure performed by neurosurgeons in North America. Unfortunately, for a variety of reasons, the surgery-related success of such procedures is far from perfect, particularly in those patients in whom multiple lumbar procedures have been performed. ${ }^{1}$

Many different operative variables have been proposed to explain such an imperfect surgery-related outcome. Of consequence, the "timed force" exerted against the involved nerve root as it is retracted during decompressive surgery has attracted considerable attention..$^{1-3,5}$ Our study was designed to focus on the dynamics of such retraction.

\section{CLINICAL MATERIAL AND METHODS}

In an observational controlled setting, we conducted a prospective analysis of 20 patients undergoing first-time microdiscectomy for single-level disc herniation. The cohort consisted of 12 men and eight women who ranged in age from 21 to 65 years (mean $42.25 \pm 15$ years). During surgery, all patients were placed in a prone position on a Jackson spinal table. In all patients, intraoperative EMG monitoring was performed in the dermatomal area subserved by the involved nerve root. A specially engineered Love nerve root retractor (Codman, Piscataway, NJ) was constructed and a sensor attached to measure the retraction force applied (Fig. 1).

The nerve root retractor was used to alter the position of the involved nerve root intraoperatively, thereby optimiz-

Abbreviation used in this paper: $\mathrm{EMG}=$ electromyography. ing the surgical exposure. Efforts were made to minimize the actual time of nerve root retraction. Measurements were obtained during maximal and minimal retraction of the involved nerve root and length of time were recorded. All patients underwent clinical assessment in the immediate postoperative period and at a minimum 1 year following surgery.

\section{RESULTS}

The degree of pressure was initially demarcated in grams of force and converted to newtons $(1 \mathrm{~N}=101.74 \mathrm{~g}$ of force). The mean force applied to the compromised nerve root in our study group was $6.95 \pm 1.2 \mathrm{~N}$, which is equivalent to $710.01 \pm 21.3 \mathrm{~g}$ of force. In our study population the mean retraction time was $39.5 \pm 21$ seconds. No intraoperative EMG-documented irritation was noted during retraction.

No patient in our study group experienced motor dysfunction in the immediate postoperative period. In the immediate postoperative period, four patients, however, did experience sensory changes in the dermatomal area subserved by the decompressed nerve root, although by the time of discharge these deficits resolved. In one of the two patients in whom a mean force between 200 and $399 \mathrm{~g}$ was applied to the compromised nerve root sensory changes developed. Of the five patients in whom the mean applied force was between 400 and $600 \mathrm{~g}$, sensory changes developed in one. Of the eight patients in whom the mean applied force was 600 to $799 \mathrm{~g}$ two experienced sensory changes. In the six patients subjected to 800 to $999 \mathrm{~g}$ of force, however, no sensory changes were ob- 


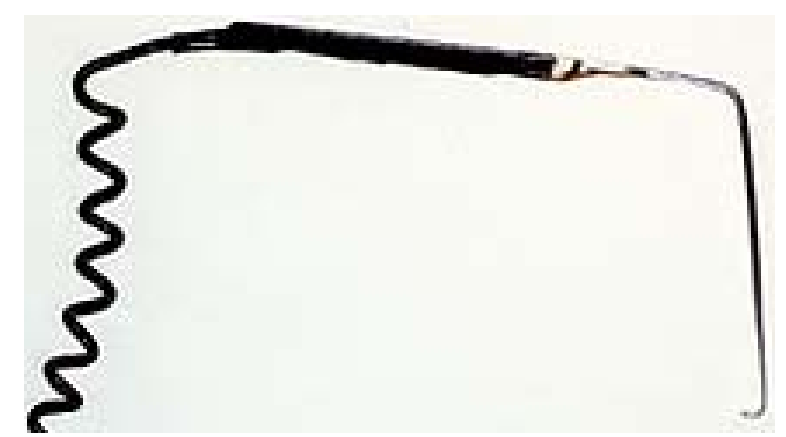

Fig. 1. Photograph demonstrating the modified Love nerve root retractor with sensor.

served. Thus, we found that with minimal retraction time no obvious correlation exists between the intraoperative retractive force applied to a compromised nerve root and postoperative sensory changes (Fig. 2).

Moreover at 1-year follow up no patient was left with any obvious neurological sequelae regardless of the retraction force was applied during operative intervention.

\section{DISCUSSION}

An important variable in determining surgery-related outcome in patients undergoing lumbar discectomy is the presurgical force dynamics directed against a compromised nerve root. In 1999, Takahashi, et al., ${ }^{6}$ measured the existing pressures between the herniated disc and the affected nerve root in patients undergoing actual lumbar surgery. Nerve root pressures ranged from 7 to $256 \mathrm{~mm}$ $\mathrm{Hg}$. These pressures were measured after laminotomy, with the patient in the prone position, before proceeding with the discectomy. ${ }^{6}$ Surprisingly, the amount of nerve root pressure, although correlated with the severity of preoperative neurological deficits, did not correlate with the sensitivity of preoperative straight leg test. Unfortunately, no outcome analysis was performed to document the postoperative impact of this variable.

The additive force of surgical retraction may further damage an already compromised nerve root. Logically increased discal impingement against a compromised nerve root should be an important variable in demarcating preoperative clinical findings and possibly postoperative outcome. In their pioneering worked published in 1984, Spencer and colleagues ${ }^{4}$ established in human cadaver studies the amount of lumbar nerve root retraction force necessary to obtain surgical exposure. Nagayama and colleagues ${ }^{2}$ demonstrated in rats that excessive retraction of lumbar nerve roots caused postoperative nerve root damage that could be observed in postoperative anatomical studies. ${ }^{2}$

Matsui and colleagues ${ }^{1}$ published a study of 31 patients undergoing lumbar discectomy; they described the intraoperative nerve root retraction pressure and corresponding perfusion of the compromised nerve root. The authors found that the length of time in which elevated nerve root pressure was applied produced an additional effect that increased postoperative neurological compromise. In all patients in whom retraction force exceeded $170 \mathrm{~g} / \mathrm{ml}$ for longer than 14 minutes, neurological deficit was present postoperatively. Thus, they advocated minimizing nerve root retraction as much as possible.

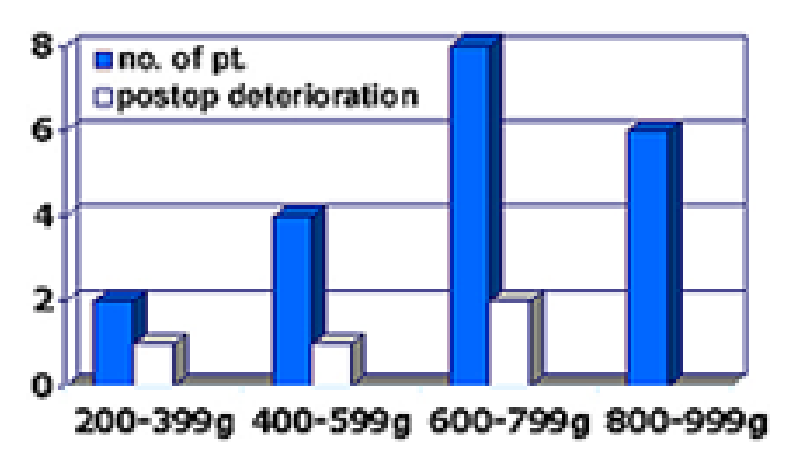

Fig. 2. Bar graph demonstrating correlation between retraction force applied and postoperative sensory changes. $\mathrm{pt}=$ patients.

Intuitively, the findings reported by Matsui and colleagues ${ }^{1}$ seem reasonable. One would expect that untoward nerve root pressure would increase the likelihood of transient or permanent neurological deficits. Surprisingly, this was not the case in our study. We did not document any particular adverse outcome in the immediate or longterm follow-up period in patients subjected to nerve root retraction forces much greater than those applied in the study by Matsui and colleagues. The explanation for this variance likely resides in our much shorter period of applied retraction force. The additional impact of prolonged nerve retraction was thus avoided. By extrapolation, our findings suggest that in cases in which prolonged nerve root retraction is necessary, episodically minimizing such retraction may diminish postoperative difficulty.

\section{CONCLUSIONS}

We found, in lumbar discectomy, that routine and sometimes substantial nerve root retraction applied during intermittent short periods of time did not cause nerve root irritation, documented on intraoperative EMG monitoring and reflected by clinical outcome.

\section{References}

1. Matsui H, Kitagawa H, Kawaguchi Y, et al: Physiologic changes of nerve root during posterior lumbar discectomy. Spine 20: 654-659, 1995

2. Nagayama R, Nakamura H, Yamano Y, et al: An experimental study of the effects of nerve root retraction on the posterior ramus. Spine 25:418-424, 2000

3. Olmarker K, Holm S, Rosenqvist AL, et al: Experimental nerve root compression. A model of acute, graded compression of the porcine cauda equina and an analysis of neural and vascular anatomy. Spine 16:61-69, 1991

4. Spencer DL, Miller JA, Bertolini JE: The effect of intervertebral disc space narrowing on the contact force between the nerve root and a simulated disc protrusion. Spine 9:422-426, 1984

5. Styf J, Willen J: The effects of external compression by three different retractors on pressure in the erector spine muscles during and after posterior lumbar spine surgery in humans. Spine 23:354-358, 1998

6. Takahashi K, Shima I, Porter RW: Nerve root pressure in lumbar disc herniation. Spine 24:2003-2006, 1999

Manuscript received June 17, 2002.

Accepted in final form July 3, 2002.

Address reprint requests to: Carlos Feltes, M.D., Georgia Neurosurgical Institute, 840 Pine Street Suite 880, Macon, Georgia 31201. email: feltesmd@cs.com. 